\title{
Fast magnetosonic wave excitation by an array of wires with time-modulated currents
}

\author{
G. Sanchez-Arriaga and J. R. Sanmartin \\ Escuela Técnica Superior de Ingenieros Aeronáuticos, Universidad Politécnica de Madrid, Madrid, Spain \\ Received: 7 October 2009 - Revised: 26 January 2010 - Accepted: 1 February 2010 - Published: 18 February 2010
}

\begin{abstract}
The excitation of Fast Magnetosonic (FMS) waves by a cylindrical array of parallel tethers carrying timemodulated current is discussed. The tethers would fly vertical in the equatorial plane, which is perpendicular to the geomagnetic field when its tilt is ignored, and would be stabilized by the gravity gradient. The tether array would radiate a single FMS wave. In the time-dependent background made of geomagnetic field plus radiated wave, plasma FMS perturbations are excited in the array vicinity through a parametric instability. The growth rate is estimated by truncating the evolution equation for FMS perturbations to the two azimuthal modes of lowest order. Design parameters such as tether length and number, required power and mass are discussed for Low Earth Orbit conditions. The array-attached wave structure would have the radiated wave controlled by the intensity and modulation frequency of the currents, making an active experiment on non-linear low frequency waves possible in real space plasma conditions.
\end{abstract}

Keywords. Space plasma physics (Wave-wave interactions)

\section{Introduction}

Nonlinear magnetohydrodynamic (MHD) wavefronts at celestial bodies moving through magnetized plasmas are common in space; the "Alfvén wings" observed at Jupiter's satellite Io (Acuna and Ness, 1981) or the cometary waves at Giacobini-Zinner and Halley (Brinca, 1997) are just two examples. The generation of an artificial wavefront in the whistler frequency range by using a planar array of electrodynamic tethers in Low Earth Orbit (LEO) was proposed in recent work (Sanchez-Arriaga and Sanmartín, 2010). In the

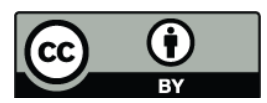

Correspondence to: G. Sanchez-Arriaga (gonzalo.sanchez@upm.es) suggested experiment, a power supply drives a time modulated current along the tethers acting as an antenna system, which radiates a single circularly-polarized whistler wave. A parametric instability, due to pumping by the time-modulated background made of geomagnetic field and radiated wave, gives rise to two unstable coupled whistler perturbations, leading to the onset of a nonlinear wavefront. With both intensity and frequency of the radiated wave controlled, this boundary-free experiment would allow studying nonlinear wave interactions and turbulence in real space plasmas.

A similar parametric instability in the near field of a MHD wave radiated by an appropriate array of tethers will be here used to generate a wavefront with frequency below the ion cyclotron frequency $\Omega_{\mathrm{i}}$. A cylindrical array of parallel tethers with its axis normal to the ambient magnetic field $B_{0}$ is shown to radiate a single FMS in the cold plasma approximation. Although both Alfvén and FMS MHD modes could be parametrically coupled to the radiated wave, we will here consider the growth rate for just FMS excitation. For typical LEO conditions ( $B_{0} \approx 0.3 \mathrm{G}$, plasma density $n_{0} \approx 10^{6} \mathrm{~cm}^{-3}$, Alfvén velocity $V_{\mathrm{A}} \approx 163 \mathrm{~km} \mathrm{~s}^{-1}$ and $\mathrm{O}^{+}$ions) the frequency and wavelength of the radiated wave would be below $\Omega_{\mathrm{i}} \approx$ $180 \mathrm{rad} \mathrm{s}^{-1}$ and above $\lambda=2 \pi V_{\mathrm{A}} / \Omega_{\mathrm{i}} \approx 5.7 \mathrm{~km}$, respectively.

This first analysis will focus on the parametric instability basics, although other relevant issues such as system mass and power efficiency will be considered too. The equipment required for wave detection could follow the lines of satellite ACTIVNY (1990), which is an example of a scientific experiment on low frequency in-situ emission (Molchanov et al., 1997). Similarly to the ACTIVNY case, subsatellites for wave detection would be necessary. We note however that ACTIVNY used a pulsed transmission mode whereas in our suggested experiment power supply would be continuously on so as to generate a wavefront stationary in the tether frame.

In-situ wave emission by orbiting tethers was considered for steady currents (Drell et al., 1965; Barnett and Olbert, 
1986; Estes, 1988; Sanmartin and Martinez-Sanchez, 1995; Sanmartin and Estes, 1997), and recently for modulated currents, in a Radiation Belt Remediation scheme (Inan et al., 2003). In a RBR mission, as in our experiment, required lengths make use of rigid antennas impossible, (flexible) tethers becoming the only feasible solution. Further, a tether is typically arranged to exchange current with the ambient plasma through end contactors, allowing the extraordinary simplification of exhibiting current uniform throughout its length, as opposed to a regular antenna, where current must vanish at its ends. Tether array systems have been also discussed for electric solar sailing (Janhunen, 2004). An updated overview about science applications of electrodynamic tethers can be found in work by Sanmartin (2009).

Our analysis is focused on a region close to the tethered structure, where the radiated wave gives rise to a parametric instability usually known as magnetoacoustic pumping (Vahala and Montgomery, 1971). The general formalism for a uniform pumped wave was first introduced by Nishikawa (1968). The parametric instability, has been predicted by Vahala and Montgomery (1971), before being observed in the laboratory (Lehane and Paoloni, 1972). The theoretical model was extended to include Hall and resistivity terms (Cramer, 1975) and boundary effects (Cramer and Sy, 1979). Dusty and multicomponent plasmas have been studied too (Hertzberg et al., 2003, 2004).

The intensity of the pumped waves in the experiment is both distance and azimuthal-angle dependent. The problem thus belongs to the theory of parametric instabilities in inhomogeneous plasmas (Perkins and Flick, 1971; Rosenbluth, 1972; Liu et al., 1973; Afeyan and Williams, 1997). Instead of using the formal WKB method or a variational approach, we roughly estimate the growth rate by truncating the equation for the perturbations to the two lowest azimuthal modes. The dependence on distance $r$ to the array is handled first by looking for an approximate resonance-like condition between radial wave-vectors of the modes and then by radial averaging.

A few waves, those with largest growth rate in the parametric instability analysis, may dominate the dynamics during the early nonlinear stage. Truncation models of the Derivative nonlinear Schrödinger equation (DNLS) (Rogister, 1971) and the triple- degenerate DNLS (TDNLS) (Hada, 1993) have been proposed as a starting point to investigate a Hall-MHD nonlinear wave-front (Sanmartin et al., 2004; Sanchez-Arriaga et al., 2007, 2009a, b). Since our experiment would happen in a non-uniform plasma and the propagation is not quasi-parallel to the ambient magnetic field, alternatives to the DNLS and the TDNLS equations must be looked for.

The paper is organized as follows. In Sect. 2 the cylindrical configuration of tethers is justified and the mathematical model is presented. The perturbation scheme involves waves of two disparate magnitude: the radiated wave and the perturbations. The first one, a cylindrical FMS wave, is calculated in Sect. 3 where we also show that no Alfvén wave is radiated. The Mathieu-like equation that governs the dynamic of just FMS perturbations is derived in Sect. 4 and the growth is determined in Sect. 5. Characteristic values of relevant parameters, as well as a trade-off of growth rate versus system mass and radiation efficiency, are discussed in Sect. 6. Conclusions are finally given in Sect. 7.

\section{General considerations}

\subsection{Cylindrical configuration of tethers}

The planar array of tethers previously proposed to excite whistler waves is not an adequate configuration for the MHD frequency domain in two respects. Whistler wavelengths in LEO are of order of hundreds of meters while in the case of MHD waves they would be of order of kilometres to tens of kilometres. This makes the planar array configuration, which required a large number of tethers, unrealistically heavy here. Further, and more important, the selected tether-array geometry should be such as to radiate a single MHD mode, for a simpler mathematical analysis.

We consider an array of $N$ tethers carrying a uniform current $I_{1}$ on the surface of a cylinder of radius $R \ll \lambda_{1}$ (wavelength of radiated wave), with its axis normal to the ambient magnetic field $B_{0}$; for $N=10$, say, the magnetic field from the active array is already nearly azimuthal close to it, with $\theta$ in Fig. 1 the azimuth. Further, our linearized analysis will require the array-generated magnetic field at its surface to be much less than $B_{0}$ (a condition later shown to exclude the case of a single tether),

$\frac{\mu_{0} N I_{1}}{2 \pi R} \ll B_{0}$

Figure 1 shows the cylindrical array. The ambient magnetic field is taken perpendicular to the tethers and a set of cylindrical coordinates $(r, \theta)$ is used in the following sections. These conditions can be attained in equatorial orbit, with the geomagnetic field horizontal if ignoring its tilt, and the tethers flying vertical in the orbital plane. The gravity gradient makes the array attitude stable (Arnold, 1987).

\subsection{Mathematical model}

The present analysis assumes several simplifying hypothesis. First, the spatial dependence of the wave fields is kept two-dimensional by assuming a tether length $L$ much larger than the wavelength $\lambda_{1}$ of the radiated wave, and a current distribution uniform along the tethers

$\boldsymbol{I}=I_{1} \cos \Omega_{1} t \boldsymbol{u}_{z}$

Uniform current distributions were considered in the past for radiation impedance considerations (Hastings and Wang, 1987; Hastings et al., 1988), and require end plasma contactors with short enough time response. 


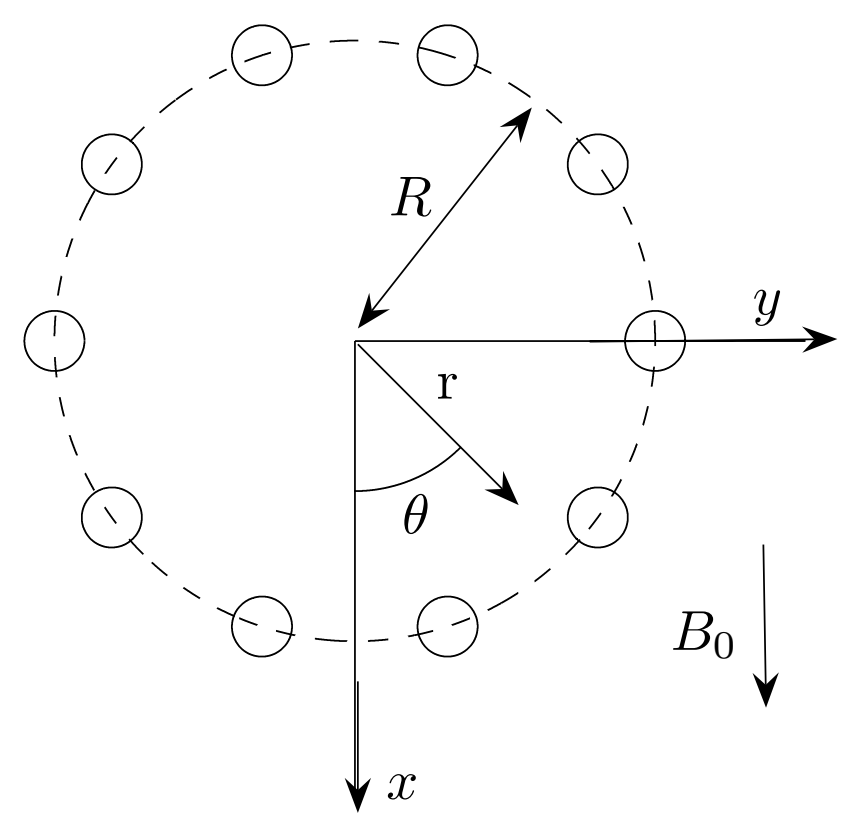

Fig. 1. Cylindrical configuration of tethers; $\theta$ will be called azimuthal angle.

Secondly, the modulation frequency $\Omega_{1}$ will be well below the ion cyclotron frequency $\Omega_{1} \ll \Omega_{i}$ and the plasma will be assumed cold. Hence, Hall term and coupling between Alfvén and Fastmagnetosonic (FMS) modes will be neglected. Finally the plasma is assumed at rest in the tether frame because orbital and Alfvén velocities $V_{0}$ and $V_{\mathrm{A}}$ satisfy $V_{0} \ll V_{\mathrm{A}}$, the relative velocity thus introducing a small frequency shift.

We will use the cold MHD plasma model equations,

$\frac{\partial \rho}{\partial t}+V_{\mathrm{A}} \nabla \cdot(\rho \boldsymbol{v})=0$

$\rho\left[\frac{\partial \boldsymbol{v}}{\partial t}+V_{\mathrm{A}}(\boldsymbol{v} \cdot \nabla) \boldsymbol{v}\right]=V_{\mathrm{A}}(\nabla \times \boldsymbol{B}) \times \boldsymbol{B}$

$\frac{\partial \boldsymbol{B}}{\partial t}-\frac{\eta}{\mu_{0}} \nabla^{2} \boldsymbol{B}=V_{\mathrm{A}} \nabla \times(\boldsymbol{v} \times \boldsymbol{B})$

where $\eta$ is the plasma resistivity, and ambient values for density $\rho_{0}$, magnetic field $B_{0}$ and Alfvén velocity $V_{\mathrm{A}}$ were used to construct dimensionless variables $\rho, \boldsymbol{B}$ and $\boldsymbol{v}$. Equations (3-5) will be solved using the Ampere law (Eq. 1) for the cylinder array as boundary condition,

$\boldsymbol{B}(r=R)=\boldsymbol{u}_{\boldsymbol{x}}+\frac{\mu_{0} N I_{1}}{2 \pi R B_{0}} \cos \Omega_{1} t \boldsymbol{u}_{\theta}$

Following Vahala and Montgomery (1971), the dependent variables are written as made of three contributions of disparate magnitude, in a double independent linearization

$\rho=1+\varepsilon \rho_{1}+\rho^{1}$

$$
\begin{aligned}
& v=\varepsilon v_{1}+v^{1} \\
& B=u_{x}+\varepsilon B_{1}+B^{1}
\end{aligned}
$$

where $\varepsilon$ is a small parameter that helps keeping track of terms of equal order. In Eqs. (7a-c), magnitudes with subscript 1 correspond to the radiated wave while superscript 1 represents any plasma perturbation. Variables with superscript 1 are much smaller than products of $\varepsilon$ and subscript 1 variables. Terms quadratic, say, in the driven fields, in effect quadratic in $\varepsilon$, will be ignored because they represent pure forcing terms, which can be verified not to contribute to a parametric instability.

\section{Radiated wave}

The radiated wave is calculated by substituting expansion (7) in Eqs. (3-5) and collecting terms of order $\varepsilon$

$$
\begin{aligned}
& \frac{\partial \rho_{1}}{\partial t}+V_{\mathrm{A}} \nabla \cdot \boldsymbol{v}_{\mathbf{1}}=0 \\
& \begin{aligned}
& \frac{\partial \boldsymbol{v}_{\mathbf{1}}}{\partial t}=V_{\mathrm{A}}\left(\nabla \times \boldsymbol{B}_{\mathbf{1}}\right) \times \boldsymbol{u}_{\boldsymbol{x}} \\
& \frac{\partial \boldsymbol{B}_{1}}{\partial t}-\frac{\eta}{\mu_{0}} \nabla^{2} \boldsymbol{B}_{\mathbf{1}}=V_{\mathrm{A}} \nabla \times\left(\boldsymbol{v}_{\mathbf{1}} \times \boldsymbol{u}_{\boldsymbol{x}}\right) \\
&=V_{\mathrm{A}}\left[\frac{\partial \boldsymbol{v}_{\mathbf{1}}}{\partial x}-\left(\nabla \cdot \boldsymbol{v}_{1}\right) \boldsymbol{u}_{x}\right]
\end{aligned}
\end{aligned}
$$

The continuity equation is decoupled from Eqs. (9) and (10); also, Eq. (9) shows $v_{x 1}=0$. Linear system (8-10) contains both Alfvén and FMS modes, which will be now studied separately.

The FMS mode can be analyzed combining the divergence of Eq. (9)

$\frac{\partial}{\partial t}\left(\nabla \cdot \boldsymbol{v}_{1}\right)=-V_{\mathrm{A}} \nabla^{2} B_{x 1}$

with the $\boldsymbol{u}_{\boldsymbol{x}}$ component of Eq. (10), giving the characteristic equation

$$
\left[\frac{\partial^{2}}{\partial t^{2}}-V_{\mathrm{A}}^{2}\left(1+\frac{\eta}{\mu_{0} V_{\mathrm{A}}^{2}} \frac{\partial}{\partial t}\right) \nabla^{2}\right] B_{x 1}=0 .
$$

Substituting a solution of the form $B_{x 1}=f(r) \sin \theta e^{-i \Omega_{1} t}$ in Eq. (12) yields the first order Bessel equation

$$
\left[1+\frac{V_{\mathrm{A}}^{2}}{\Omega_{1}^{2}}\left(1-\frac{i \eta \Omega_{1}}{\mu_{0} V_{\mathrm{A}}^{2}}\right)\left(\frac{d^{2}}{d r^{2}}+\frac{1}{r} \frac{d}{d r}-\frac{1}{r^{2}}\right)\right] f=0
$$

the solutions being the Bessel functions $J_{1}$ and $Y_{1}$ with argument $\xi \equiv k_{1} r$ and wavevector

$$
k_{1}= \pm \frac{\Omega_{1}}{V_{\mathrm{A}}} \frac{1}{\sqrt{1-\frac{i \eta \Omega_{1}}{\mu_{0} V_{\mathrm{A}}^{2}}}} \approx \pm \frac{\Omega_{1}}{V_{\mathrm{A}}}\left(1+\frac{i}{2} \frac{\eta \Omega_{1}}{\mu_{0} V_{\mathrm{A}}^{2}}\right)
$$


where $\eta \Omega_{1} / \mu_{0} V_{\mathrm{A}}^{2} \ll 1$ was assumed. As we will see, the radiation condition now implies that the actual solution for $f$ must be proportional to the Hankel function of the first kind, $H_{1}^{(1)}=J_{1}+i Y_{1}$. Equations (8-10) next show $\rho_{1}=B_{x 1}$, $\boldsymbol{v}_{\mathbf{1}}=v_{y 1} \boldsymbol{u}_{\boldsymbol{y}}$, and $\boldsymbol{B}_{1}=\boldsymbol{B}_{\theta} \boldsymbol{u}_{\boldsymbol{\theta}}$; we absorb any constant factor into parameter $\varepsilon$. The full radiated wave solution is finally given as

$\rho_{1}=-\operatorname{Re}\left[i H_{1}^{(1)}(\xi) \sin \theta e^{-i \Omega_{1} t}\right]$

$\boldsymbol{v}_{\mathbf{1}}=-\operatorname{Re}\left(H_{0}^{(1)}(\xi) e^{-i \Omega_{1} t}\right) \boldsymbol{u}_{\boldsymbol{y}}$

$\boldsymbol{B}_{\mathbf{1}}=\operatorname{Re}\left[i H_{1}^{(1)}(\xi) e^{-i \Omega_{1} t}\right] \boldsymbol{u}_{\boldsymbol{\theta}}$

with $\varepsilon$ in expansion (7) determined by using boundary condition (6) with $\xi_{\mathrm{R}} \equiv k_{1} R \ll 1, H_{1}^{(1)} \approx-2 i / \pi k_{1} R$, yielding both $\varepsilon$ and full solution independent of $R$ for $k_{1} R$ small enough,

$\varepsilon \approx \frac{\mu_{0} N I_{1} k_{1}}{4 B_{0}}$.

For $\xi \gg 1$, the Hankel functions become

$H_{n}^{(1)}(\xi) \approx \sqrt{\frac{2}{\pi \xi}} e^{i\left(\xi-\frac{n \pi}{2}-\frac{\pi}{4}\right)}$

requiring use of the plus sign in Eq. (14). As advanced the radiation condition can be verified on the time-averaged Poynting vector far away from the tether, reading in dimensional form

$<\boldsymbol{S}>=-\frac{\varepsilon^{2} V_{\mathrm{A}} B_{0}^{2}}{\mu_{0}}<\left(\boldsymbol{v}_{\mathbf{1}} \times \boldsymbol{u}_{\boldsymbol{x}}\right) \times \boldsymbol{B}_{1}>=\frac{\varepsilon^{2} V_{\mathrm{A}} B_{0}^{2}}{\pi \mu_{0} k_{1} r} \boldsymbol{u}_{\boldsymbol{r}}$

To end the discussion about the FMS wave, we estimate the total power radiated by the tether array by integrating the Poynting flux vector on a cylindrical surface of radius $r$ and length $L$

$W_{\mathrm{R}} \sim \frac{2 \varepsilon^{2} L V_{\mathrm{A}} B_{0}^{2}}{\mu_{0} k_{1}}=\frac{\mu_{0} \Omega_{1} L N^{2} I_{1}^{2}}{8}$

The Alfvén mode could be similarly analyzed in terms of components of normalized current density $\boldsymbol{j} \equiv \nabla \times \boldsymbol{B}$ and vorticity $\boldsymbol{w} \equiv \nabla \times \boldsymbol{v}$ along the ambient magnetic field $\boldsymbol{B}_{0}$, as opposed to velocity divergence and magnetic field along $\boldsymbol{B}_{0}$ for the FMS mode (Cramer, 2001). Taking the curl of Eqs. (9) and (10) and projecting on $\boldsymbol{u}_{x}$ yield

$\frac{\partial w_{x 1}}{\partial t}=V_{\mathrm{A}} \frac{\partial j_{x 1}}{\partial x}$

$\frac{\partial j_{x 1}}{\partial t}=V_{\mathrm{A}} \frac{\partial w_{x 1}}{\partial x}$

Equations (22) and (23) and boundary condition (6) show that no Alfvén wave is radiated by the system. Therefore, within the cold MHD approximation and assuming a purely azimuthal magnetic field at $r=R$ as given by Eq. (6), the array of tethers will just radiate a cylindrical FMS wave.

\section{Alfvén and Fastmagnetosonic perturbations}

We next derive evolution equations for FMS and Alfvén mode perturbations. Expansion (7) is again substituted in Eqs. (3-5) and terms proportional to the excited (superscript 1) waves and the product of excited waves and components of the radiated (driven) wave with subscript 1 are collected. For simplicity, we now neglect the resistivity, which would just modify the growth rate slightly. The set of expanded equations then reads

$\frac{\partial \rho^{1}}{\partial t}+V_{\mathrm{A}} \nabla \cdot \boldsymbol{v}^{1}=-\varepsilon V_{\mathrm{A}} \nabla \cdot\left(\rho_{1} \boldsymbol{v}^{1}+\rho^{1} \boldsymbol{v}_{1}\right)$

$$
\begin{aligned}
\frac{\partial \boldsymbol{v}^{1}}{\partial t}-V_{\mathrm{A}} \boldsymbol{j}^{1} \times \boldsymbol{u}_{\boldsymbol{x}}= & \varepsilon\left\{V _ { \mathrm { A } } \left[\boldsymbol{j}_{1} \times \boldsymbol{B}^{1}+\boldsymbol{j}^{1} \times \boldsymbol{B}_{1}-\left(\boldsymbol{v}_{1} \cdot \nabla\right) \boldsymbol{v}^{1}\right.\right. \\
& \left.\left.-\left(\boldsymbol{v}^{1} \cdot \nabla\right) \boldsymbol{v}_{1}\right]-\rho_{1} \frac{\partial \boldsymbol{v}^{1}}{\partial t}-\rho^{1} \frac{\partial \boldsymbol{v}_{1}}{\partial t}\right\}(25)
\end{aligned}
$$

$\frac{\partial \boldsymbol{B}^{1}}{\partial t}-V_{\mathrm{A}} \nabla \times\left(\boldsymbol{v}^{1} \times \boldsymbol{u}_{x}\right)=\varepsilon V_{\mathrm{A}} \nabla \times\left[\boldsymbol{v}_{1} \times \boldsymbol{B}^{1}+\boldsymbol{v}^{1} \times \boldsymbol{B}_{1}\right](26)$

The left-hand sides Eqs. (24-26) contain the same operators of Eqs. (8-10), which would yield the dispersion relation of the Alfvén and FMS modes, while the right-hand sides represent the parametric coupling between excited waves and radiated (background) fields.

Equation (25) shows the velocity component $v_{x}^{1}$ to be of order $O(\varepsilon)$. Next, from Eq. (26) we obtain

$\frac{\partial B_{x}^{1}}{\partial t}+V_{\mathrm{A}} \psi^{1}=-\varepsilon V_{\mathrm{A}}\left[\frac{\partial}{\partial y}\left(v_{y 1} B_{x}^{1}\right)+B_{x 1} \psi^{1}+v_{y}^{1} \frac{\partial B_{x 1}}{\partial y}\right]$

where we introduced the velocity divergence (FMS) variable

$\psi^{1} \equiv \frac{\partial v_{y}^{1}}{\partial y}+\frac{\partial v_{z}^{1}}{\partial z}$

Equations (24) and (27) show that the difference $\rho^{1}-B_{x}^{1}$ is also of order $O(\varepsilon)$. Hence, terms in right-hand sides involving $v_{x}^{1}$ or $\rho^{1}-B_{x}^{1}$ can be neglected because they are $O\left(\varepsilon^{2}\right)$. This result together with Eq. (9), $\rho_{1}=B_{x 1}$ and $\boldsymbol{j}_{\mathbf{1}}=j_{1 z} \boldsymbol{u}_{z}$, make Eq. (25) read

$$
\begin{aligned}
{\left[\frac{\partial \boldsymbol{v}^{1}}{\partial t}-V_{\mathrm{A}} \boldsymbol{j}^{1} \times \boldsymbol{u}_{x}\right]=} & \frac{\varepsilon V_{\mathrm{A}}}{\left(1+\varepsilon B_{x 1}\right)}\left[\left(B_{y 1} \boldsymbol{j}^{1}+B_{y}^{1} \boldsymbol{j}_{1}\right)\right. \\
& \left.\times \boldsymbol{u}_{y}-v_{y 1} \frac{\partial \boldsymbol{v}^{1}}{\partial y}-v_{y}^{1} \frac{\partial \boldsymbol{v}_{1}}{\partial y}\right]
\end{aligned}
$$

where the term $\varepsilon B_{x 1}$ in the denominator can be now neglected.

The evolution equation of $\psi^{1}$ is obtained from Eq. (29)

$$
\begin{aligned}
& \frac{\partial \psi^{1}}{\partial t}+V_{\mathrm{A}} \nabla^{2} B_{x}^{1}= \\
& \varepsilon V_{\mathrm{A}}\left(B_{y 1} \frac{\partial j_{x}^{1}}{\partial z}-v_{y 1} \frac{\partial \psi^{1}}{\partial y}-2 \frac{\partial v_{y 1}}{\partial y} \frac{\partial v_{y}^{1}}{\partial y}-v_{y}^{1} \frac{\partial^{2} v_{y 1}}{\partial y^{2}}\right)
\end{aligned}
$$


For quasi-parallel propagation of the Alfvén mode, $j_{x}^{1}=$ $j_{x}^{1}(x, \varepsilon y, \varepsilon z)$ and $w_{x}^{1}=w_{x}^{1}(x, \varepsilon y, \varepsilon z)$, the term $\partial j_{x}^{1} / \partial z$ in Eq. (30) can be neglected and the FMS mode equation is finally obtained by combining Eqs. (27) and (30)

$$
\begin{aligned}
& \frac{\partial^{2} \psi^{1}}{\partial t^{2}}-V_{\mathrm{A}}^{2} \nabla^{2} \psi^{1}= \\
& \varepsilon V_{\mathrm{A}}\left\{V_{\mathrm{A}} \nabla^{2}\left[\frac{\partial}{\partial y}\left(v_{y 1} B_{x}^{1}\right)+B_{x 1} \psi^{1}+v_{y}^{1} \frac{\partial B_{x 1}}{\partial y}\right]\right. \\
& \left.-\frac{\partial}{\partial t}\left(v_{y 1} \frac{\partial \psi^{1}}{\partial y}+2 \frac{\partial v_{y 1}}{\partial y} \frac{\partial v_{y}^{1}}{\partial y}+v_{y}^{1} \frac{\partial^{2} v_{y 1}}{\partial y^{2}}\right)\right\}
\end{aligned}
$$

Equation (31) will be solved in the next section assuming that the perturbation $\psi^{1}$ is made of just two waves. The variable $B_{x}^{1}$ that appears on the right hand side will be obtained from Eq. (27) with $\varepsilon=0$, while the velocity component $v_{y}^{1}$ is related to $\psi^{1}$ by introducing the vorticity definition $\boldsymbol{w} \equiv \nabla \times \boldsymbol{v}$ in Eq. (28)

$$
\left(\frac{\partial^{2}}{\partial y^{2}}+\frac{\partial^{2}}{\partial z^{2}}\right) v_{y}^{1}=\frac{\partial \psi^{1}}{\partial y}-\frac{\partial w_{x}^{1}}{\partial z} \approx \frac{\partial \psi^{1}}{\partial y}
$$

To derive an Alfvén wave evolution equation for future work, first note that Eq. (31) is decoupled from the Alfvénic variables $j_{x}^{1}$ and $w_{x}^{1}$. FMS variables can thus be solved independently and then substituted in the evolution equations for $j_{x}^{1}$ and $w_{x}^{1}$, giving rise to purely forcing terms. Ignoring such terms one would finally find

$$
\frac{\partial^{2} j_{x}^{1}}{\partial t^{2}}-V_{\mathrm{A}}^{2} \frac{\partial^{2} j_{x}^{1}}{\partial x^{2}}=\varepsilon V_{\mathrm{A}}^{2} \frac{\partial}{\partial x}\left(j_{x}^{1} \frac{\partial B_{y 1}}{\partial y}+B_{x 1} \frac{\partial j_{x}^{1}}{\partial x}-2 w_{x}^{1} \frac{\partial v_{y 1}}{\partial y}\right)
$$

\section{Dispersion relation}

Equation (31) describes the FMS wave evolution. The driven fields on the right hand side depend on distance $r$ and angle $\theta$, and an infinite number of radial and azimuthal modes are thus coupled together. An estimation of the growth rate of the FMS mode can be obtained, however, by keeping the two azimuthal modes of lowest order in an expansion of the solution

$\psi^{1}=\cos k_{z} z\left[\psi_{a} J_{0}\left(k_{a} r\right) e^{-i \omega_{a} t}+\psi_{b} J_{1}\left(k_{b} r\right) \sin \theta e^{-i \omega_{b} t}\right]+\mathrm{cc}$

As usual, cc denotes complex conjugated and frequencies $\omega_{a, b}$ are related by the resonance condition $\omega_{a}+\omega_{b}^{*}=\Omega_{1}$. For convenience we will use the dimensionless parameters $\bar{\omega}_{a, b} \equiv \omega_{a, b} / \Omega_{1}, \bar{k}_{a, b} \equiv k_{a, b} / k_{1}, \bar{k}_{z} \equiv k_{z} / k_{1}$.

Before finding the growth rate, we note that the resonance condition restricts the admissible value of $\bar{k}_{a}$ and $\bar{k}_{b}$. At $\varepsilon=$

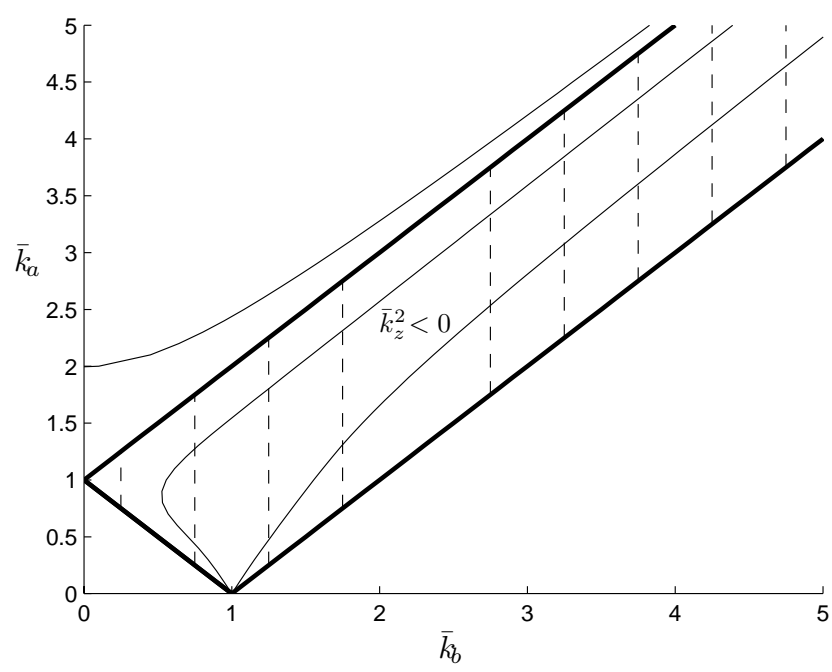

Fig. 2. Thick solid lines show the allowable domain $\bar{k}_{z}^{2}>0$. Thin solid lines are solutions of Eq. (42).

0 one has $\bar{\omega}_{a, b 0}= \pm \sqrt{\bar{k}_{a, b}^{2}+\bar{k}_{z}^{2}}$ and the resonance condition yields a relation between wavevectors

$\bar{k}_{z}^{2}=\left(\frac{1-\bar{k}_{a}^{2}-\bar{k}_{b}^{2}}{2}\right)^{2}-\bar{k}_{a}^{2} \bar{k}_{b}^{2}$

Frequencies $\bar{\omega}_{a, b}$ at $\varepsilon=0$ read then $\bar{\omega}_{a 0}=\left(1+\bar{k}_{a}^{2}-\bar{k}_{b}^{2}\right) / 2$ and $\bar{\omega}_{b 0}=\left(1+\bar{k}_{b}^{2}-\bar{k}_{a}^{2}\right) / 2$. Setting the right hand side of Eq. (35) equal to zero, one finds

$\bar{k}_{b}^{2}=\left(1 \pm \bar{k}_{a}\right)^{2}$

defining a region in the plane $\bar{k}_{a}-\bar{k}_{b}$ where $\bar{k}_{z}$ is real (see Fig. 2).

The derivation of the growth rate requires the fields $B_{x}^{1}$ and $v_{y}^{1}$ that appears on the RHS of Eq. (31). The magnetic field $B_{x}^{1}$ is directly found from Eq. (27)

$B_{x}^{1}=-i V_{\mathrm{A}} \cos k_{z} z\left[\frac{\psi_{a}}{\omega_{a}} J_{0}\left(k_{a} r\right) e^{-i \omega_{a} t}+\frac{\psi_{b}}{\omega_{b}} J_{1}\left(k_{b} r\right) \sin \theta e^{-i \omega_{b} t}\right]$ $+c c$.

As regards the velocity $v_{y}^{1}$ we write it as

$$
\begin{aligned}
v_{y}^{1}= & \frac{\cos k_{z} z}{k_{1}}\left[C_{a} \psi_{a} J_{1}\left(k_{a} r\right) \sin \theta e^{-i \omega_{a} t}+C_{b} \psi_{b} J_{0}\left(k_{b} r\right) e^{-i \omega_{b} t}\right] \\
& +c c
\end{aligned}
$$

with coefficients $C_{a}=4 \bar{k}_{a} /\left(4 \bar{k}_{z}^{2}+3 \bar{k}_{a}^{2}\right)$ and $C_{b}=$ $-\bar{k}_{b} /\left(2 \bar{k}_{z}^{2}+\bar{k}_{b}^{2}\right)$ obtained by inserting Eqs. (34) and (38) in Eq. (32) (see Appendix for auxiliary calculations).

Expressions (34), (37) and (38) are finally substituted in Eq. (31). Collecting terms with the same azimuthal dependence yields two equations for the FMS mode amplitudes $\psi_{a}$ 
and $\psi_{b}^{*}$. The wave $\psi_{a}\left(\psi_{b}^{*}\right)$ in the left hand side of Eq. (31) is coupled with the product between a driven term proportional to $e^{-i \Omega_{1} t}\left(e^{i \Omega_{1} t}\right)$ and $\psi_{b}^{*}\left(\psi_{a}\right)$ in the right hand side. A tedious but straightforward calculation would give

$$
\begin{aligned}
& {\left[\bar{\omega}_{a}^{2}-\left(\bar{k}_{a}^{2}+\bar{k}_{z}^{2}\right)\right] J_{0}\left(\bar{k}_{a} \xi\right) \psi_{a} \approx-\varepsilon g_{0}(\xi) \psi_{b}^{*}} \\
& {\left[\bar{\omega}_{b}^{* 2}-\left(\bar{k}_{b}^{2}+\bar{k}_{z}^{2}\right)\right] J_{1}\left(\bar{k}_{b} \xi\right) \psi_{b}^{*} \approx-\varepsilon g_{1}(\xi) \psi_{a}}
\end{aligned}
$$

the $g_{0,1}$ functions being (see Appendix)

$$
\begin{aligned}
& \frac{8}{i} g_{0} \equiv \bar{k}_{b}\left[\frac{2 \kappa^{4}+\kappa^{3}+7 \kappa^{2}+7 \kappa-1-4 \bar{k}_{a}\left(\kappa^{2}+3\right)}{(1-\kappa)\left(\kappa^{2}+1-2 \bar{k}_{a}^{2}\right)} H_{0} J_{0}\right. \\
& \left.-\frac{2\left(2 H_{0} J_{2}-\bar{k}_{b} H_{1} J_{3}\right)}{1-\kappa}-2 H_{2} J_{2}\right] \\
& -\frac{1}{2} \frac{\kappa^{5}-\kappa^{4}-12 \kappa^{3}-12 \kappa^{2}+11 \kappa+5-2 \bar{k}_{a}^{2}\left(\kappa^{3}-9 \kappa^{2}-17 \kappa+9+12 \bar{k}_{a}^{2}\right)}{(1-\kappa)\left(\kappa^{2}+1-2 \bar{k}_{a}^{2}\right)} H_{1} J_{1}
\end{aligned}
$$

$$
\begin{aligned}
& \frac{8}{i} g_{1} \equiv\left[\frac{\left(\kappa^{2}+2 \kappa+5\right)(1-\kappa)}{(1+\kappa)}+\frac{4 \bar{k}_{a}^{2}\left(3 \kappa^{2}+\kappa-2\left(1+\bar{k}_{a}^{2}\right)\right)}{(1+\kappa)\left[(1+\kappa)^{2}-\bar{k}_{a}^{2}\right]}\right] H_{1}^{*} J_{0} \\
& +2 \bar{k}_{a}\left[\frac{2 \kappa^{2}+6}{1+\kappa}-\frac{7+\kappa^{2}}{(1+\kappa)^{2}-\bar{k}_{a}^{2}}\right] H_{0}^{*} J_{1} \\
& -\frac{4 \bar{k}_{a}^{2} H_{3}^{*} J_{2}}{(1+\kappa)^{2}-\bar{k}_{a}^{2}} \\
& +\frac{\left[5 \kappa^{3}+\kappa^{2}+7 \kappa+3+4 \bar{k}_{b}^{2}(1-\kappa)\right] \bar{k}_{a} H_{2}^{*} J_{1}-2 \bar{k}_{a}^{2}\left(5 \kappa^{2}-1-4 \bar{k}_{b}^{2}\right) H_{1}^{*} J_{2}}{(1+\kappa)\left[(1+\kappa)^{2}-\bar{k}_{a}^{2}\right]}
\end{aligned}
$$

where we defined

$\kappa \equiv \bar{k}_{a}^{2}-\bar{k}_{b}^{2}$

Note that the argument of the Hankel functions is just $\xi$, whereas the argument of $J_{n}$ Bessel functions in $g_{0}\left(g_{1}\right)$ is $\bar{k}_{b} \xi\left(\bar{k}_{a} \xi\right)$.

There is no way for ratios $g_{0} / J_{0}$ and $g_{1} / J_{1}$ to be $\xi$ independent as required by the homogeneous system (39a), (39b). In the following, we solve for a dispersion relation by radial averaging both equations from $\xi_{\mathrm{R}}$ to $\infty$. We first, however, look for a resonance-like condition $\bar{k}_{b}\left(\bar{k}_{a}\right)$ that avoids rapid radial variations in the above ratios. Both, left and right hand sides of Eqs. (39a) and (39b) do decay as $\xi \rightarrow \infty$, as $\propto 1 / \sqrt{\xi}$ and $\propto 1 / \xi$ respectively. Also, for $\xi \rightarrow \xi_{\mathrm{R}} \ll 1$, the ratio $g_{0} / J_{0}$ in Eq. (39a) behaves as $\ln \xi$, which may be considered $O(1)$ as usual in asymptotic analyses. On the other hand, the ratio $g_{1} / J_{1}$ in Eq. (39b) would in principle behave as $1 / \xi^{2}$ due to the $H_{1}^{*} J_{0}, H_{2}^{*} J_{1}$ and $H_{3}^{*} J_{2}$ terms. This disagreement is dealt with by requiring those three terms to

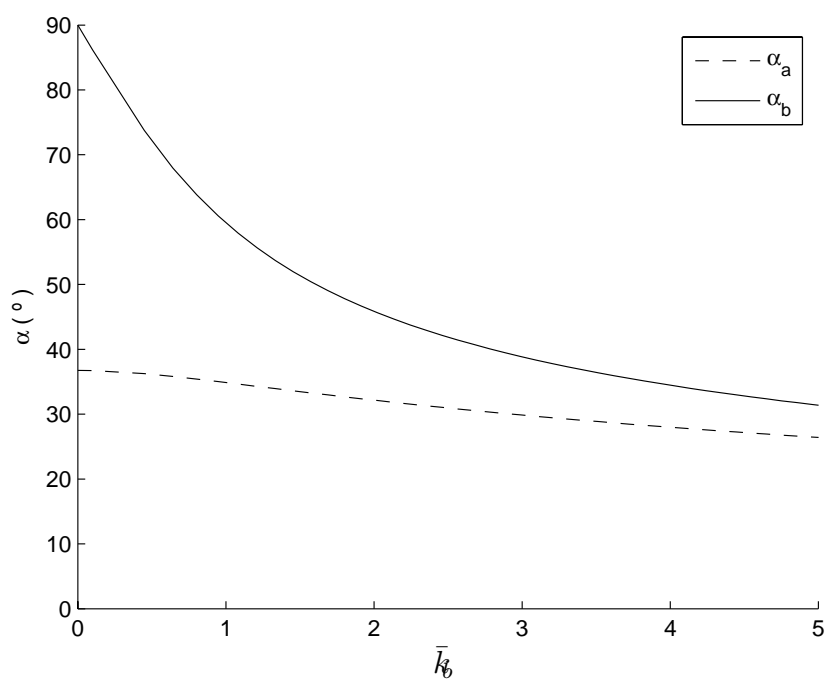

Fig. 3. Angles $\alpha_{a}$ and $\alpha_{b}$ versus normalized wave-vector $\bar{k}_{b}$.

balance out at small $\xi$, leading to a wave-vector resonance condition $\bar{k}_{b}\left(\bar{k}_{a}\right)$,

$$
\begin{gathered}
(1+\kappa)\left[\left(\kappa^{2}+2 \kappa+5\right)\left(1-\kappa^{2}\right)-8 \bar{k}_{a}^{4}\right] \\
+2 \bar{k}_{a}^{2}\left(3 \kappa^{3}+9 \kappa^{2}+5 \kappa-5\right)=0
\end{gathered}
$$

with $\kappa$ given by Eq. (41). The truncation in Eq. (34) would thus be strongly invalid unless wavevectors $\bar{k}_{a}$ and $\bar{k}_{b}$ satisfy Eq. (42).

Figure 2 summarizes the above results involving wavevectors $\bar{k}_{a}$ and $\bar{k}_{b}$. The thick solid lines correspond to $\bar{k}_{z}$ vanishing in Eq. (36). Within the enclosed (shaded) region $\bar{k}_{z}$ is imaginary. The thin solid lines correspond to the solutions of Eq. (42) and only the higher line makes $\bar{k}_{z}$ real. As $\bar{k}_{a} \rightarrow \infty$, Eq. (42) yields $\kappa / \bar{k}_{a} \rightarrow 2$, and then Eq. (41) gives $\bar{k}_{a}-\bar{k}_{b} \approx 1$, which can be verified in Fig. 2. Equation (35) shows $k_{z} / k_{a} \rightarrow 0$ as $\bar{k}_{a} \rightarrow \infty$.

The angles $\alpha_{a} \equiv \arctan \left(k_{z} / k_{a}\right)$ and $\alpha_{b} \equiv \arctan \left(k_{z} / k_{b}\right)$ are plotted versus the normalized wave-vector $\bar{k}_{b}$ in Fig. 3. Angle $\alpha_{a}$, with values around $35^{\circ}$, is nearly insensitive to $\bar{k}_{b}$ for the range considered, as opposed to $\alpha_{b}$; hence, the $\theta$-independent wave labelled $a$, appears nearly confined to a conic surface with axis parallel to the array at angle $\sim 35^{\circ}$. Actually, both $\alpha_{a}$ and $\alpha_{b}$ vanish with $k_{z} / k_{a}$ as $\bar{k}_{a} \rightarrow \infty$. Also, for a properly selected $k_{1}$, too large values of $\bar{k}_{a}$ and $\bar{k}_{b}$ would place $k_{a}$ and $k_{b}$ wave-vectors beyond the MHD regime.

Next, we integrate Eqs. (39a) and (39b) from $\xi_{R}$ and $\infty$, leading to

$$
\begin{aligned}
& {\left[\bar{\omega}_{a}^{2}-\left(\bar{k}_{a}^{2}+\bar{k}_{z}^{2}\right)\right] \psi_{a} \approx-\varepsilon C_{0} \psi_{b}^{*}} \\
& {\left[\bar{\omega}_{b}^{* 2}-\left(\bar{k}_{b}^{2}+\bar{k}_{z}^{2}\right)\right] \psi_{b}^{*} \approx-\varepsilon C_{1} \psi_{a}}
\end{aligned}
$$


where

$C_{0,1}=\frac{\int_{\xi_{\mathrm{R}}}^{\infty} g_{0,1} d \xi}{\int_{\xi_{\mathrm{R}}}^{\infty} J_{0,1} d \xi} \approx \bar{k}_{a, b} \int_{\xi_{\mathrm{R}}}^{\infty} g_{0,1} d \xi$

Equations $(43 \mathrm{a}, \mathrm{b})$ yield the dispersion relation

$\left[\bar{\omega}_{a}^{2}-\left(\bar{k}_{a}^{2}+\bar{k}_{z}^{2}\right)\right]\left[\bar{\omega}_{b}^{* 2}-\left(\bar{k}_{b}^{2}+\bar{k}_{z}^{2}\right)\right]=\varepsilon^{2} C_{0} C_{1}$

We now introduce the dimensionless frequency shift $\widetilde{\omega}$ and growth rate $\tilde{\gamma}$ defined by $\bar{\omega}_{a} \equiv \bar{\omega}_{a 0}+\varepsilon(\widetilde{\omega}+i \tilde{\gamma})$ and $\bar{\omega}_{b}^{*} \equiv$ $\bar{\omega}_{b 0}-\varepsilon(\widetilde{\omega}+i \tilde{\gamma})$. Using Eq. (18), the actual growth rate is

$\gamma=\tilde{\gamma} \frac{\mu_{0} N I_{1} k_{1}}{4 B_{0}} \Omega_{1}$

The dispersion relation now reads

$(\tilde{\omega}+i \tilde{\gamma})=\sqrt{\frac{-C_{0} C_{1}}{1-\kappa^{2}}}$

where we used the approximations $\bar{\omega}_{a}^{2}-\left(\bar{k}_{a}^{2}+\bar{k}_{z}^{2}\right) \approx$ $2 \bar{\omega}_{a 0} \varepsilon(\widetilde{\omega}+i \tilde{\gamma})$ and $\bar{\omega}_{b}^{* 2}-\left(\bar{k}_{b}^{2}+\bar{k}_{z}^{2}\right) \approx-2 \bar{\omega}_{b 0} \varepsilon(\widetilde{\omega}+i \tilde{\gamma})$.

Figure 4 shows the dimensionless growth rate $\tilde{\gamma}$ versus angle $\alpha_{b}$ for two different values of $\xi_{\mathrm{R}}$. At $\alpha_{b}=90^{\circ}$, corresponding to $\bar{k}_{a} \approx 2.0, \bar{k}_{b}=0$, the growth rate vanishes because $C_{1}=0$ in Eq. (44). The growth rate monotonically increases when the angle $\alpha_{b}$ decreases, a situation corresponding with a larger wave-vector (see Fig. 3). The parametric instability becomes stronger because the perturbation wavelengths are shorter and a higher number of spatial oscillations interact with the radiated wave, which also decays with distance to the tethers. At $\alpha_{b} \approx 60^{\circ}$, which corresponds to $\bar{k}_{b} \approx 1$, the growth rate would actually diverge. This is because the integral $C_{0}$ contain integrands of the type $\cos ^{2} \xi / \xi$ which make $C_{0}$ to diverge logarithmically with the upper limit of the integral. This has not been resolved numerically in Fig. 4. The local maximum of $\tilde{\gamma}$ will be actually limited by the imaginary part of $k_{1}$ in Eq. (14) up to now neglected. The peak would thus be logarithmically large $\tilde{\gamma} \approx \ln \left(2 \mu_{0} V_{\mathrm{A}} / \eta k_{1}\right)$.

\section{Discussion}

In addition to the growth rate, there are other important parameters in the experiment such as the characteristic tether length, the mass of the system, or the orbit. An equatorial orbit with the tethers flying vertical and the geomagnetic field normal to the array would be a suitable configuration. System mass considerations as regards altitude will be shown to involve both plasma density and characteristic ion mass.

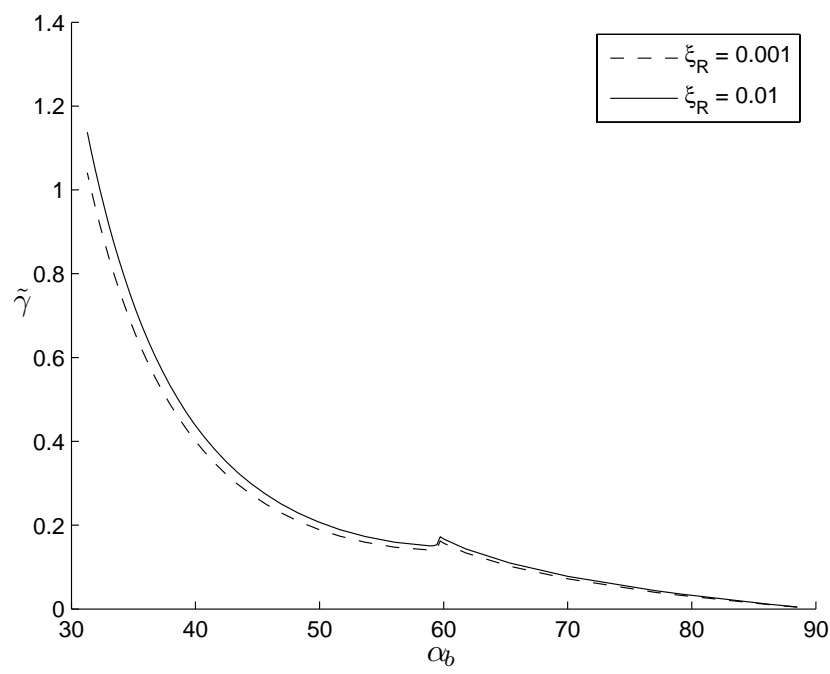

Fig. 4. Dimensionless growth rate versus angle $\alpha_{b}$.

System mass for the experiment would basically be made of tether hardware and a power subsystem,

$$
\begin{aligned}
M_{\mathrm{S}} & =M_{\mathrm{t}}+\alpha W_{\mathrm{Ohm}}\left(1+\frac{W_{\mathrm{R}}}{W_{\mathrm{Ohm}}}\right) \\
& \approx\left[A_{\mathrm{t}} \rho+\frac{\alpha I_{1}^{2}}{\sigma_{\mathrm{c}} A_{\mathrm{t}}}\left(1+\frac{N \mu_{0} \Omega_{1} \sigma_{\mathrm{c}} A_{\mathrm{t}}}{8}\right)\right] N L
\end{aligned}
$$

where $\alpha$ is the inverse specific power of the power supply while $A_{\mathrm{t}}, \rho$ and $\sigma_{\mathrm{c}}$ are tether cross section, density and conductivity respectively. The radiated power $W_{\mathrm{R}}$ was taken from Eq. (21). Fixing all parameters except area $A_{\mathrm{t}}$, a $M_{\mathrm{S}}$ minimum is reached at

$A_{\mathrm{t}}=I_{1} \sqrt{\alpha / \sigma_{\mathrm{c}} \rho}$

yielding

$\left.M_{\mathrm{S}}\right|_{\min }=2 \rho N A_{\mathrm{t}} L$

where we neglected the ratio $W_{\mathrm{R}} / W_{\mathrm{Ohm}}$, assumed small. From Eqs. (46) and (49) we find

$\gamma=\tilde{\gamma} \Omega_{1} \frac{\pi}{2} \frac{\mu_{0}}{B_{0}} \sqrt{\frac{\sigma_{\mathrm{c}} \rho}{\alpha}} \frac{N A_{\mathrm{t}}}{\lambda_{1}}$

allowing to write

$\left.M_{\mathrm{S}}\right|_{\min }=\frac{4}{\pi \widetilde{\gamma}} \frac{B_{0}}{\mu_{0}} \sqrt{\frac{\alpha \rho}{\sigma_{\mathrm{c}}}} \frac{L \lambda_{1}}{\Omega_{1}} \gamma$

Also, using Eq. (50) in the expression for the power ratio in Eq. (48), we find

$\frac{W_{\mathrm{R}}}{W_{\mathrm{Ohm}}}=\left.\frac{\mu_{0} \sigma_{\mathrm{c}}}{16 \rho L} \Omega_{1} M_{\mathrm{S}}\right|_{\min }$.

In our analysis we assumed $\lambda_{1} / L$ and $\Omega_{1} / \Omega_{\mathrm{i}}$ small to allow a two-dimensional description of the radiated wave and our 
ignoring the Hall term. Here, just for a mass estimate, we set $L=\lambda_{1}$ and $\Omega_{1}=2 \pi V_{\mathrm{A}} / \lambda_{1}=\Omega_{\mathrm{i}}$ while using $\tilde{\gamma}$ as found previously, yielding in Eq. (52)

$$
\left.M_{\mathrm{S}}\right|_{\min }=\frac{16 \pi}{\tilde{\gamma} \mu_{0}^{2} e^{3}} \sqrt{\frac{\alpha \rho}{\sigma_{\mathrm{c}}}} \frac{m_{i}^{2}}{n_{0}} \gamma
$$

with similar simplifications in Eqs. (51) and (53). We note that $\left.M_{\mathrm{S}}\right|_{\min }$ does not depend on the geomagnetic field, and is proportional to both $m_{i}^{2} / n_{0}$ and growth rate. Hence, experiments in LEO and MEO would actually require a similar system mass because the density drop would be compensated by the characteristic ion changing from $\mathrm{O}^{+}$to $\mathrm{H}^{+}$.

For tether values $\rho=2.7 \times 10^{3} \mathrm{~kg} \mathrm{~m}^{-3}$ and $\sigma_{\mathrm{c}}=3.5 \times$ $10^{7} \mathrm{Ohm}^{-1} \mathrm{~m}^{-1}$, state-of-the-art $\alpha=20 \mathrm{~kg} \mathrm{KW}^{-1}$, and the typical LEO conditions $\left(B_{0} \approx 0.3 \mathrm{G}, n_{0} \approx 10^{6} \mathrm{~cm}^{-3}\right.$ and $\mathrm{O}^{+}$ ions, $V_{\mathrm{A}} \approx 163 \mathrm{~km} \mathrm{~s}^{-1}$ and $\Omega_{\mathrm{i}} \approx 180 \mathrm{rad} \mathrm{s}^{-1}$ ), taking representative value $\tilde{\gamma} \approx 0.3$ in Fig. 4 and growth rate $\gamma=$ $0.015 \mathrm{~s}^{-1}$ yields $\left.M_{\mathrm{S}}\right|_{\min } \approx 340 \mathrm{~kg}$ in Eq. (54). With $L=$ $\lambda_{1}=5.7 \mathrm{~km}$, we also find $W_{\mathrm{R}} / W_{\mathrm{Ohm}} \approx 0.01 \mathrm{in} \mathrm{Eq.} \mathrm{(53),} \mathrm{small}$ as previously assumed, and $N A_{\mathrm{t}} \approx 11 \mathrm{~mm}^{2}$ in Eq. (50), and then $N I_{1} \approx 24 \mathrm{~A}$ in Eq. (49).

To simplify the analysis, we took the number of tethers $N$ and the radius $R$ of the cylindrical array as satisfying two conditions: first, the magnetic field was approximated as purely azimuthal at $r=R \ll \lambda_{1}$, and secondly we assumed $\mu_{0} N I_{1} / 2 \pi R B_{0}$ small to allow a linear analysis. For the value $N I_{1}=24 \mathrm{~A}$, we find $\mu_{0} N I_{1} / 2 \pi R B_{0} \approx 0.157 \mathrm{~m} / \mathrm{R}$. This makes impossible the use of a single tether and suggests setting the array radius at a few meters. Note that the actual value of $R$ would enter the solution nowhere else, in case of $k_{1} R$ small enough. On the other hand, a value $N=10$, say, would yield a magnetic field already nearly azimuthal close to the array. Hence, a set of tethers with fixed connections and flying as a single spacecraft would be a possible configuration.

\section{Conclusions}

The generation of a FMS wave-front using an electrodynamic tether array, similarly to a previous analysis for whistler waves (Sanchez-Arriaga and Sanmartin, 2010), could be used to investigate nonlinear wave interactions and turbulence in space plasmas. Such experiment would have several qualities such as in situ wave emission in natural plasmas, boundary free propagation conditions and an active character because the frequency and the intensity of the FMS radiated wave would be controlled by the power supply. This first work analyses the parametric instability that should produce a saturated nonlinear stage in a frame moving with the array.

The radiated FMS wave is parametrically coupled to two cylindrical FMS perturbations waves. One of them, which is independent of the azimuthal angle is only excited if its wave-vector lies close to a conic surface with angle away from the array normal about $35^{\circ}$; the second parametrically coupled wave covers a broad angle range.

To keep the problem analytically tractable, we made several hypotheses that we now revisit. Variations along the tether axis direction were neglected requiring tether lengths much longer than the typical wavelength and uniform current distributions along the tethers. As showed in Sect. 5, the first hypothesis yields an unrealistically heavy system while the second one could be addressed with appropriate tether plasma contactors. Both hypotheses might be removed in numerical calculations by considering a fully 3-D radiated wave. For simplicity the Hall term was also ignored but there is no reason to keep the driven frequency well below $\Omega_{i}$ in a real experiment.

Future works may also consider other aspects that are not directly related with the parametric instability. We cite the dynamical response of the contactors to exchange current with the plasma, a detailed stability analysis of the cylindrical configuration including forces among tethers, wave detection and scientific missions. All of them would be important for a mission to generate an artificial nonlinear MHD wavefront in space plasmas.

\section{Appendix A}

\section{Growth rate calculation}

In this appendix we summarize some auxiliary steps for the calculation of the growth rate. To simplify the notation the superscript 1 and the argument $\xi$ in any Hankel function will be omitted. Note that Bessel functions multiplying $\psi_{a}\left(\psi_{b}\right)$ has argument $\bar{k}_{a} \xi\left(\bar{k}_{b} \xi\right)$.

The coefficients $C_{a}$ and $C_{b}$ that appear in the velocity $v_{y}^{1}$ are found taking into account

$$
\begin{aligned}
\frac{\partial \psi^{1}}{\partial y}= & -\frac{\cos k_{z} z}{2} \\
& {\left[2 \psi_{a} k_{a} J_{1} \sin \theta e^{-i \omega_{a} t}-\psi_{b}^{*} k_{b}\left(J_{0}+J_{2} \cos \right.\right.} \\
\frac{\partial^{2} v_{y}^{1}}{\partial y^{2}}= & \frac{-\cos k_{z} z}{2 k_{1}} \\
& {\left[\frac{C_{a} \psi_{a} k_{a}^{2}}{2}\left(3 J_{1} \sin \theta+J_{3} \sin 3 \theta\right) e^{-i \omega_{a} t}\right.} \\
& \left.+C_{b} \psi_{b}^{*} k_{b}^{2}\left(J_{0}-J_{2} \cos 2 \theta\right) e^{i \omega_{b}^{*} t}\right]+\mathrm{cc}
\end{aligned}
$$$$
\left[2 \psi_{a} k_{a} J_{1} \sin \theta e^{-i \omega_{a} t}-\psi_{b}^{*} k_{b}\left(J_{0}+J_{2} \cos 2 \theta\right) e^{i \omega_{b}^{*} t}\right]+\mathrm{cc}
$$

and $\partial^{2} v_{y}^{1} / \partial z^{2}=-k_{z}^{2} v_{y}^{1}$.

The calculus of the functions $g_{0,1}$ is carried out with the following auxiliary operations:

$$
\frac{\partial}{\partial y}\left[\nabla^{2}\left(v_{y 1} B_{x}^{1}\right)\right] \approx \frac{i k_{1}^{2}}{2}\left\{\frac { F _ { a } } { \overline { \omega } _ { a 0 } } \left[\bar{k}_{T a}^{2}\left(H_{1}^{*} J_{0}+\bar{k}_{a} H_{0}^{*} J_{1}\right)\right.\right.
$$




$$
\begin{aligned}
& \left.+\bar{k}_{a}\left(\bar{k}_{a} H_{1}^{*}\left(J_{0}-J_{2}\right)+\left(H_{0}^{*}-H_{2}^{*}\right) J_{1}\right)\right] \\
& +\frac{F_{b}}{4 \bar{\omega}_{b 0}}\left[2 \bar{k}_{T b}^{2}\left(\bar{k}_{b} H_{0} J_{0}-H_{1} J_{1}\right)\right. \\
& \left.\left.+\bar{k}_{b}\left(\bar{k}_{b} H_{1}\left(J_{3}-3 J_{1}\right)+2 H_{0}\left(J_{0}-J_{2}\right)\right)\right]\right\}
\end{aligned}
$$

$$
\begin{aligned}
\nabla^{2}\left(B_{x 1} \chi^{1}\right) \approx & -\frac{i k_{1}^{2}}{4}\left\{2 F_{a}\left[\bar{k}_{a}\left(H_{0}^{*}-H_{2}^{*}\right) J_{1}+\bar{k}_{T a}^{2} H_{1}^{*} J_{0}\right]\right. \\
& \left.+F_{b}\left[\bar{k}_{b}\left(H_{0} J_{0}+H_{2} J_{2}\right)-\bar{k}_{T b}^{2} H_{1} J_{1}\right]\right\}
\end{aligned}
$$

$$
\begin{aligned}
\nabla^{2}\left(\frac{\partial B_{x 1}}{\partial y} v_{y}^{1}\right) \approx & \frac{-i k_{1}^{2}}{8}\left\{F _ { a } C _ { a } \left[\bar{k}_{a}\left(3 H_{1}^{*} J_{0}-2 H_{1}^{*} J_{2}+H_{3}^{*} J_{2}\right)\right.\right. \\
& \left.+\bar{k}_{T a}^{2}\left(2 H_{0}^{*}-H_{2}^{*}\right) J_{1}\right] \\
& \left.+2 F_{b} C_{b}\left[2 \bar{k}_{b} H_{1} J_{1}-\bar{k}_{T b}^{2} H_{0} J_{0}\right]\right\}
\end{aligned}
$$

$$
\frac{\partial}{\partial t}\left(v_{y}^{1} \frac{\partial \chi^{1}}{\partial y}\right) \approx \frac{i V_{\mathrm{A}} k_{1}^{2}}{4}\left(2 \bar{\omega}_{b 0} \bar{k}_{a} H_{0}^{*} J_{1} F_{a}+\bar{\omega}_{a 0} \bar{k}_{b} H_{0} J_{0} F_{b}\right)(\mathrm{A} 6)
$$

$$
\begin{aligned}
\frac{\partial}{\partial t}\left(\frac{\partial v_{y 1}}{\partial y} \frac{\partial v_{y}^{1}}{\partial y}\right) \approx & \frac{i V_{\mathrm{A}} k_{1}^{2}}{8}\left(\bar{\omega}_{b 0} \bar{k}_{a} C_{a} H_{1}^{*}\left(2 J_{0}-J_{2}\right) F_{a}\right. \\
& \left.+2 \bar{\omega}_{a 0} \bar{k}_{b} C_{b} H_{1} J_{1} F_{b}\right)
\end{aligned}
$$

$$
\begin{aligned}
\frac{\partial}{\partial t}\left(v_{y}^{1} \frac{\partial^{2} v_{y 1}}{\partial y^{2}}\right) \approx & \frac{i V_{\mathrm{A}} k_{1}^{2}}{8}\left(\bar{\omega}_{b 0} C_{a}\left(2 H_{0}^{*}-H_{2}^{*}\right) J_{1} F_{a}\right. \\
& \left.-2 \bar{\omega}_{a 0} C_{b} H_{0} J_{0} F_{b}\right)
\end{aligned}
$$

where the symbol $\approx$ was used to denote higher order azimuthal modes, e.g. terms proportional to $\cos 2 \theta$, were neglected. We also defined

$F_{a} \equiv \psi_{a} \cos \left(k_{z} z\right) \sin \theta e^{i \omega_{b}^{*} t}$

$F_{b} \equiv \psi_{b}^{*} \cos \left(k_{z} z\right) e^{-i \omega_{a} t}$

$\bar{k}_{T a, b}^{2} \equiv 1+\bar{k}_{z}^{2}+\bar{k}_{a, b}^{2}$

Substituting the above expressions in Eq. (31) and using Eq. (35) to eliminate $\bar{k}_{z}$ yield $g_{0,1}$.

Acknowledgements. This work was supported by the Ministry of Science and Technology of Spain under Grant No. ESP2004-01511.

The authors thank P. Janhunen for his assistance in evaluating this paper and fruitful comments.

Topical Editor R. Nakamura thanks P. Janhunen for his help in evaluating this paper.

\section{References}

Acuna, M. H. and Ness, F. M.: Standing Alfvén wave current system at Io: Voyager I Observations, J. Geophys. Res., 86, 85138521, 1981.

Afeyan, B. B. and Williams, E. A.: A variational approach to parametric instabilities in inhomogeneous plasmas I: Two model problems, Phys. Plasmas, 4(11), 3788-3802, 1997.

Arnold, D. A.: The behavior of long tethers in space, The Journal of Astronautical Sciences, 35(1), 3-18, 1987.

Barnett, A. and Olbert, S.: Radiation of plasma waves by a conducting body moving through magnetized plasma, J. Geophys. Res., 91, 10117-10135, 1986.

Brinca, A. L.: Generation and nonlinear evolution of cometary waves. Nonlinear Waves and Chaos in Space Plasmas, edited by: Hada, T. and Matsumoto, H., Terrapub, Tokio, 45-76 1997.

Cramer, N. F.: Parametric excitation of ion-cyclotron waves, Plasma Phys., 17, 967-972, 1975.

Cramer, N. F. and Sy, W. N-C.: Parametric decay of magnetoacoustic oscillations in a cylindrical plasma, J. Plasma Phys., 22, 549-562, 1979.

Cramer, N. F.: The Physics of Alfvén Waves, WILEY-VCH Verlag Berlin GmbH, 2001.

Drell, S. D., Foley, H. M., and Ruderman, M. A.: Drag and propulsion of large satellite in the ionosphere: An Alfvén propulsion engine in space, J. Geophys. Res., 70, 3131-3145, 1965.

Estes, R. D.: Alfvén waves from an electrodynamic tethered satellite system, J. Geophys. Res., 93, 945-956, 1988.

Hada, T.: Evolution of large amplitude alfvén waves in the solar wind with $\beta \sim 1$, J. Geophys. Res. Lett., 20(22), 2415-2418, 1993.

Hastings, D. E. and Wang, J.: The radiation impedance of an electrodynamic tether with end connectors, J. Geophys. Res. Lett., 14, 519-522, 1987.

Hastings, D. E., Barnett, A., and Olbert, S.: Radiation from large space structures in low Earth orbit with induced alternating current, J. Geophys. Res., 93, 1945-1960, 1988.

Hertzberg, M. P., Cramer, N. F., and Vladimirov, S. V.: Parametric instabilities of Alfvén waves in a dusty plasma, Phys. Plasmas, 10(8), 3160-3167, 2003.

Hertzberg, M. P., Cramer, N. F., and Vladimirov, S. V.: Parametric instabilities in a magnetized multicomponents plasmas, Phys Rev. E, 69, 056402, 2004.

Inan, U. S., Bell, T. F., Bortnik, J., and Albert, J. M.: Controlled precipitation of radiation belt electrons, J. Geophys. Res., 108(A5), 1186, doi:10.1029/2002JA009580, 2003.

Janhunen, P.: Electric sail for spacecraft propulsion, J. Propulsion Power, 20(4), 763-764, 2004.

Lehane, J. A. and Paoloni, F. J.: Parametric excitation of Alfvén waves, Plasma Physics, 14, 461-471, 1972.

Liu, C. S., Rosenbluth, M. N., and White, R. B.: Parametric scattering instabilities in inhomogeneous plasma, Phys. Rev. Lett., 31(11), 697-700, 1973.

Molchanov, O. A., Mogilevsky, M. M., Afonin, V. V., Klos, Z., Hayakawa, M., and Shima, N.: Nonlinear ELV-VLF effect observed on ACTIVNY satellite, Nonlinear Waves and Chaos in Space Plasmas, edited by: Hada, T. and Matsumoto, H., Terrapub, 1997.

Nishikawa, K.: Parametric excitation of coupled waves I. General formulation, J. Phys. Soc. Jpn, 24(4), 916-922, 1968. 
Perkins, F. W. and Flick, J.: Parametric instabilities in inhomogeneous plasmas, Phys. Fluids, 14(9), 2012, doi:10.1063/1.1693711, 1971.

Rogister, A.: Parallel Propagation of Nonlinear Low-Frequency Waves in High- $\beta$ Plasma, Phys. Fluids, 14(12), 2733-2739, 1971.

Rosenbluth, M. N.: Parametric instabilities in inhomogeneous media, Phys. Rev. Lett, 29(9), 565-567, 1972.

Sanchez-Arriaga, G., Sanmartín, J., and Elaskar, S. A.: Damping models in the truncated derivative nonlinear Schrödinger equation, Phys. Plasmas, 14, 082108, 2007.

Sanchez-Arriaga, G., Hada, T., and Nariyuki, Y.: The truncation model of the derivative nonlinear Schrödinger equation, Phys. Plasmas, 16, 042302, 2009a.

Sanchez-Arriaga, G., Hada, T., and Nariyuki, Y.: Truncation model in the Triple-degenerate derivative nonlinear Schrödinger equation, Phys. Plasmas, 16, 042303, 2009b.
Sanchez-Arriaga, G. and Sanmartin, J.: Magnetic pumping of whistler waves by tether current modulation, J. Geophys. Res., 115, A02311, doi:10.1029/2009JA014478, 2010.

Sanmartin, J. and Martinez-Sanchez, M.: The radiation impedance of orbiting conductors, J. Geophys. Res., 100, 1677-1686, 1995.

Sanmartín, J. and Estes, R. D.: Alfvén wave far field from steadycurrent tethers, J. Geophys Res., 102(A7), 14625-14630, 1997.

Sanmartin, J. R., Lopez-Rebollal O., Del Rio, E., and Elaskar, S.: Hard transition to chaotic dynamics in Alfvén wave fronts, Phys. Plasmas, 11, 2026-2035, 2004.

Sanmartin, J. R.: A Review of electrodynamic tethers for science applications, Plasma Sources Sci. Technol., in press, 2010.

Vahala, G. and Montgomery, D.: Parametric amplification of Alfvén waves, Phys. Fluids, 14(6), 1137-1140, 1971. 